\title{
Holey-Gold Films on Molybdenum Grids for Cryogenic Electron Microscopy Imaging of 2D Polymer Crystals
}

Xi Jiang ${ }^{1}$, Sunting Xuan ${ }^{2}$, Ronald Zuckermann ${ }^{2}$, Robert Glaeser ${ }^{3}$ and Nitash Balsara $^{4}$

${ }^{1}$ Materials Sciences Division, Lawrence Berkeley National Laboratory, United States, ${ }^{2}$ Molecular Foundry, Lawrence Berkeley National Laboratory, United States, ${ }^{3}$ Lawrence Berkeley National Laboratory, United States, ${ }^{4}$ University of California, Berkeley, United States

Images obtained by cryo-EM are also affected by the nature of the support on which the specimens reside. The support affects imaging due to the fact that beam-induced motion becomes a greater problem when crinkling of specimens occurs as they are cooled. Several early studies have investigated the effect that the geometry and materials-composition of the specimen support has on beam-induced motion. [1-3] Holey carbon films are commonly used in cryo-EM studies, but specimens supported on them suffer from significant beam-induced motion. Russo and Passmore demonstrated that an all-gold supporting grid (UltrAuFoil grid) was able to significantly reduce beam-induced motion in dose-fractionation movies, up to 50-fold along the $z$ (vertical) direction and twofold in the $x$-y direction, as compared to a holey carbon film on a copper grid.[4,5] In addition to beam-induced motion, the flatness of the specimen is an important factor that limits resolution of crystalline membranes composed of ordered arrays of either biological or synthetic macromolecules. [1-3,6-8] Unlike well-distributed suspensions of individual macromolecules in vitrified ice, which can be imaged without using a thin supporting film, continuous carbon films are universally used to support crystalline membranes. Carbon supporting films can, however, influence the flatness of the crystalline membrane. The crinkled specimens result in reflections in Fourier transforms of images that, in the direction perpendicular to the tilt axis, become increasingly blurred at high tilt angles. These effects dramatically reduce high-resolution information in the threedimensional (3-D) reconstructions of crystalline membranes.

We introduce a novel composite holey gold support that prevents cryo-crinkling and reduces beaminduced motion of soft specimens, building on the previously introduced all-gold support. The composite holey gold support for high-resolution cryogenic electron microscopy of soft crystalline membranes was fabricated in two steps. In the first step, a holey gold film was transferred on top of a molybdenum grid. In the second step, a continuous thin carbon film was transferred onto the holey gold film. The configuration of this grid is shown in Figures 1A to 1C. Although all of our supports for crystalline membranes also contain a continuous carbon film. We believe that the unique properties of this grid arise from the thermal expansion coefficients of the three components. The thermal expansion coefficient of carbon $\left(6 \times 10^{-60} \mathrm{C}^{-1}\right)$ is close to that of molybdenum $\left(5 \times 10^{-6}{ }^{\circ} \mathrm{C}^{-1}\right)$, while gold has a much larger thermal expansion coefficient $\left(14 \times 10^{-6}{ }^{o} \mathrm{C}^{-1}\right)$ and the thermal expansion coefficient of copper is $17 \times 10$ ${ }^{-6}{ }^{o} \mathrm{C}^{-1}$. [9] Contraction of the holey gold film is thus expected to tighten it, along with the crystalline membranes, under tension, rather than cause them to crinkle when the substrate with the specimen is cooled to low temperatures.

The samples that we used to test our grids were crystalline polypeptoid membranes. Polypeptoids are bio-inspired synthetic polymers comprising repeating $\mathrm{N}$-substituted glycine monomer units. They are similar to polypeptides except for the fact that the side chain is appended to the nitrogen atom rather than the $\alpha$-carbon. [10] Crystalline polypeptoid membranes, which are only one unit-cell thick, similar to 2-D protein membranes but with smaller unit cells, are generated by the self-assembly of di-block copolymers in dilute solutions. In a previous study, we have used images of untilted specimens to determine the structure of crystalline polypeptoid membranes with about $2 \AA$ resolution when using vitrified specimens 
prepared on a holey carbon support film. [11] Contraction of crystalline polypeptoid membrane cannot be accurately predicted since the thermal expansion coefficient of polypeptoid is unknown. However, as a synthetic polymer, the thermal expansion coefficient of polypeptoid is likely to be similar to the thermal expansion coefficients of typical semicrystalline polymers which are at least one order of magnitude larger than carbon film. In this case, the less contracted carbon film on which the crystalline polypeptoid membranes reside makes the change of lattice of membranes less than that of the free-standing membranes.

The performance of the new Au/Mo grid was characterized by quantifying beam-induced motion, flatness of specimens, and resolution. The results indicated that both the Au/Mo grids effectively reduced beam-induced motion in untilted specimens as compare to that of the holey carbon films on copper grids. $[4,5]$ The flatness of specimens was also investigated by measuring the local variation in defocus across the $45^{\circ}$ tilt images obtained from Au/Mo grid. Finally, the resolution of images of specimens tilted at about $45^{\circ}$, when supported on the Au/Mo grids, often remains about $1.5 \AA$ as shown in Figure 1D, which is better than all previous studies on crystalline polypeptoid membranes. The results thus show that these $\mathrm{Au} / \mathrm{Mo}$ grids provide excellent performance for imaging very thin crystals of a synthetic polymer.

Funding for this work was provided by the Soft Matter Electron Microscopy Program (KC11BN), supported by the Office of Science, Office of Basic Energy Science, US Department of Energy, under Contract DE-AC02-05CH11231. Work at the Molecular Foundry was supported by the Office of Science, Office of Basic Energy Sciences, of the U.S. Department of Energy under Contract No. DE-AC02$05 \mathrm{CH} 11231$.
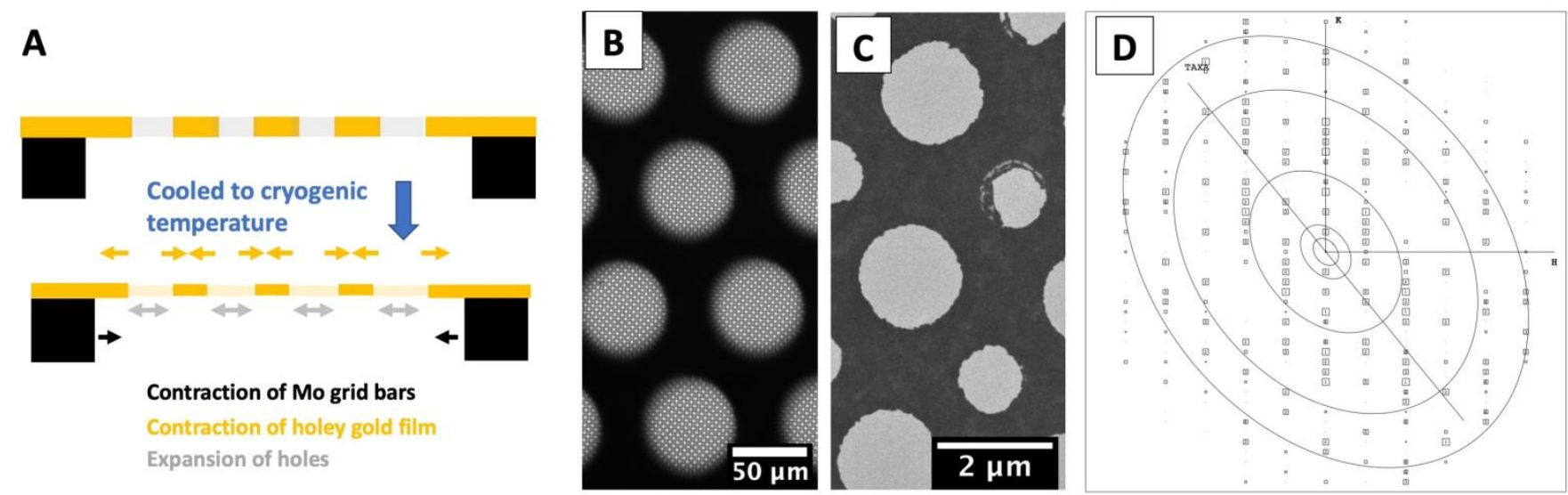

Figure 1. Figure 1. A. Schematic cartoon showing the hypothesized expansion of holes in the holey gold film due to contraction of the gold film relative to the molybdenum grid after cooling. Wrinkles in the carbon film covering the holes, which may have existed before cooling, can be flattened by increasing the size of the holes, and even the holey gold film itself may become flattened as it contracts relative to the Mo grid. Black and yellow represent molybdenum and gold, respectively. Only a few holes are shown and hole expansion is exaggerated for clarity. B. Optical microscopy image shows the morphology of holey gold film on a molybdenum grid with a thin carbon film. C. TEM micrograph shows the configuration of holes where the blue large hole is used for recording and green small hole is used for focusing in low-dose imaging mode. This image is obtained by magnifying one of the holes in B. D. The IQ plot of a specimen on $\mathrm{Au} / \mathrm{Mo}$ grid, tilted by 45o, after lattice unbending. This IQ plot is intentionally distorted here, as if it were for an untilted projection, i.e., the reciprocal lattice points are shifted to coincide with those for an untilted specimen. TAXA represents the crystal tilt axis, using the terminology in 2DX. Resolution rings are drawn in both cases at $24,12,4,2$ and $1.5 \AA$. Image quality index (IQ) $1-6$ of 
reflections in resolution ranges from zero to Nyquist (1.1 $)$ are shown as squares with correspondingly smaller size, reflecting their diminishing statistical reliability.

\section{References}

[1]. BOOY, F.P. \& PAWLEY, J.B. (1993). Cryo-Crinkling - What Happens to Carbon-Films on Copper Grids at Low-Temperature. Ultramicroscopy 48(3), 273-280.

[2]. FUJIYOSHI, Y. (1998). The structural study of membrane proteins by electron crystallography. Adv Biophys 35, 25-80.

[3]. GLAESER, R.M. (1992). Specimen Flatness of Thin Crystalline Arrays - Influence of the Substrate. Ultramicroscopy46(1-4), 33-43.

[4]. RUSSO, C.J. \& PASSMORE, L.A. (2014). Ultrastable gold substrates for electron cryomicroscopy. Science346(6215), 1377-1380.

[5]. RUSSO, C.J. \& PASSMORE, L.A. (2016). Ultrastable gold substrates: Properties of a support for high-resolution electron cryomicroscopy of biological specimens. Journal of structural biology 193(1), 33-44.

[6]. VONCK, J. (1996). A three-dimensional difference map of the $\mathrm{N}$ intermediate in the bacteriorhodopsin photocycle: Part of the F helix tilts in the $\mathrm{M}$ to $\mathrm{N}$ transition. Biochemistry 35(18), 58705878 .

[7]. VONCK, J. (2000). Parameters affecting specimen flatness of two-dimensional crystals for electron crystallography. Ultramicroscopy 85(3), 123-129.

[8]. HAN, B.G., WOLF, S.G., VONCK, J. \& GLAESER, R.M. (1994). Specimen Flatness of GlucoseEmbedded Biological-Materials for Electron Crystallography Is Affected Significantly by the Choice of Carbon Evaporation Stock. Ultramicroscopy 55(1), 1-5.

[9]. HAYNES, W.M. (2015). Handbook of Chemistry and Physics. CRC Press.

[10]. ROBERTSON, E.J., OLIVER, G.K., QIAN, M., PROULX, C., ZUCKERMANN, R.N. \& RICHMOND, G.L. (2014). Assembly and molecular order of two-dimensional peptoid nanosheets through the oil-water interface. P Natl Acad Sci USA 111(37), 13284-13289.

[11]. JIANG, X., GREER, D.R., KUNDU, J., OPHUS, C., MINOR, A.M., PRENDERGAST, D., ZUCKERMANN, R.N., BALSARA, N.P. \& DOWNING, K.H. (2018). Imaging Unstained Synthetic Polymer Crystals and Defects on Atomic Length Scales Using Cryogenic Electron Microscopy. Macromolecules 51(19), 7794-7799. 